\title{
Study on the Luminescence Properties of the Strain Compensated Quantum Well*
}

\author{
Tao Liu, Jianjun Li ${ }^{\#}$, Jiachun Li, Jun Han, Jun Deng, Linjie He, Shengjie Lin
}

Key Laboratory of Opto-Electronics Technology, Beijing University of Technology, Beijing, China. Email: liutao2011@emails.bjut.edu.cn, "lijianjun@bjut.edu.cn

Received September 2013

\begin{abstract}
Compared with the conventional strained quantum well, the InGaAs/GaAsP strain compensated quantum well (SCQW) has better optical properties, as the well layer and the barrier layer lattice mismatch with each other which results in the introduction of stress. In this paper, we adopted the Kohn-Luttinger Hamiltonian, conducted some theoretical calculations using the transfer matrix method, and finally verified the following change trend of the InGaAs quantum well following the In-group through experiments: the growth of the low In-group can improve the epitaxial flatness of the active area; the growth of the high In-group can increase the wavelength as well as the strain. In this paper, we adopted the AlGaAs barrier material instead of the GaAsP, made an analysis on the level changes of the compensation quantum well, and successfully fostered the strain compensated quantum well structure using the metal-organic chemical vapor deposition (MOCVD) system which had better optical properties compared with the strained quantum wells.
\end{abstract}

Keywords: InGaAs/AlGaAs; InGaAs/GaAsP; Strain Compensated Quantum Well; MOCVD

\section{Introduction}

InGaAs is an important semiconductor material, whose carrier mobility is more than 10 times compared with the ordinary Si material. InGaAs has many potential applications in the new generation of optical fiber communication and lasers. The high-quality strained InGaAs quantum wells have been widely used in the optoelectronic devices. The laser performance $[1,2]$ can be greatly improved by adopting the strained quantum well structure in the active layer.

When design the large optical cavity (LOC) highpower lasers, high-quality quantum wells grown is our key consideration and the design of the InGaAs/AlGaAs strained quantum wells is the focus of the design direction. However, as the critical strain thickness of the strained quantum well is small, it is difficult to release stress. In the growth process of high In composition, the lattice defects may lead to the design limitations of the long wavelength.

In the strain compensated structure, the barrier layer adopts the GaAsP which has an opposite strain with the well layer. Therefore, the above mentioned shortcomings [3] can be well overcame. Moreover, Dutta et al. [4]

\footnotetext{
*Supported by the Advanced Technology Fund of Beijing University of Technology under Grant No. 002000514312003.

\#Corresponding author.
}

found through experiments that the gain of the lasers can be improved by adopting the InGaAs/GaAsP strain compensated quantum wells instead of strained quantum wells. GaAsP and AlGaAs have a similar band gap, while GaAsP has a smaller lattice constant. The tensile stress subjected in the quantum wells compensates with the compressive resistance of the InGaAs potential well, which can greatly improve the stress properties of material growth.

By adopting the $\mathrm{In}_{0.2} \mathrm{Ga}_{0.8}$ As quantum wells which is of $8 \mathrm{~nm}$ LP-MOVCD thick growth, the AlGaAs and the GaAsP are selected as the barrier materials. Theoretical and experimental validation show that the stress of the InGaAs/AlGaAs strained quantum well grown in the GaAs substrate has increased rapidly and the critical thickness thereof has decreased rapidly. The wavelength is limited in a certain range and the long-wavelength components can't be made. The InGaAs/GaAsP strain compensated quantum wells can effectively alleviate the impact of stress. Through theoretical analysis and PL spectra measurements, the impact of the quantum wells stress on spectrum is verified.

Through theoretical analysis on the well width and the barrier material trends of the energy band structure in quantum wells, and comparative analysis of the InGaAs/ AlGaAs strained quantum well and InGaAs/GaAsP strain compensated quantum wells, we can see that compared 
with the strained quantum well, the strain compensated quantum wells have better luminescence properties.

\section{Experimental Methods}

We adopted the Emcore D125 LP-MOCVD system and conducted the epitaxial growth of the material, wherein the growth conditions are as follows: the pressure is 80 mbar; the substrate carrier speed is $1000 \mathrm{r} / \mathrm{s}$, the palladium tube purified $\mathrm{H}_{2}$ is adopted as the carrier gas; the flow rate is $30 \mathrm{slm}$, the adopted $\mathrm{V}$ group source is the high purity $\mathrm{AsH}_{3}$; the III group source are TMAl, TMGa and TMIn.

We conducted epitaxial growth in accordance with epitaxial structure in Table $\mathbf{1}$. We adopted the no-clean GaAs semi-insulating substrate. The substrate orientation is (100) biased (111) $2^{\circ}$. The grown sequence are GaAs buffer layer, n-type confinement layer, waveguide layer, quantum wells, barrier layer, quantum well, upper wave guide layer, p-cladding layer, covering layer.

As shown in Table 2, we prepared four different samples. The first two samples are InGaAs/AlGaAs strained quantum well structure. The last two samples are InGaAs/GaAsP strain compensated quantum wells. In the growth process, In with different traffic were flowed into the four samples, wherein the traffic volume are as follows: No 1:126.7 $\mathrm{cm}^{3}$; No 2: $101.7 \mathrm{~cm}^{3}$; No 3:126.7 $\mathrm{cm}^{3}$; No 4: $101.7 \mathrm{~cm}^{3}$.

Table 1. Quantum well growth parameters design.

\begin{tabular}{ccc}
\hline Layer & Composition & Width (nm) \\
\hline 1 & GaAs coating & 5 \\
2 & $\mathrm{Al}_{0.3} \mathrm{Ga}_{0.7}$ As p-cladding layer & 200 \\
3 & $\mathrm{Al}_{0.1} \mathrm{Ga}_{0.9}$ As n-waveguide layer & 200 \\
4 & barrier & 20 \\
5 & InGaAs well & 20 \\
6 & barrier & 8 \\
7 & InGaAs well & 200 \\
8 & barrier & 20 \\
9 & $\mathrm{Al}_{0.1} \mathrm{Ga}_{0.9}$ As n-waveguide layer & 200 \\
10 & $\mathrm{Al}_{0.3} \mathrm{Ga}_{0.7}$ As n-cladding layer & 300 \\
11 & $\mathrm{GaAs}$ buffer layer $_{2}$ \\
12 & GaAs substrate & 200 \\
\hline
\end{tabular}

Table 2. Barrier growth parameters of the four samples.

\begin{tabular}{ccc}
\hline Sample & In flow $\left(\mathrm{cm}^{3}\right)$ & Barrier material \\
\hline 1 & 126.7 & AlGaAs \\
2 & 101.7 & AlGaAs \\
3 & 126.7 & GaAsP \\
4 & 101.7 & GaAsP \\
\hline
\end{tabular}

\section{Theoretical Calculations}

In order to explore the stress of the quantum well, we have checked the relevant material properties [6], including the lattice constant, elastic modulus, hydrostatic pressure and shear deformation potential, etc. We have conducted some theoretical calculations by using these parameters. And then we got the change rules of the quantum well band gap following the stress, which have given the theoretical support for our experiment.

InGaAs/AlGaAs strained quantum well band gap is subject to the following three aspects:

1) The In components may result in the InGaAs material band gap changes [7];

$$
E_{g}(x)=1.424-1.614 x+0.54 x^{2}
$$

2) The thickness of the well material is thin enough to be comparable with the De Buluo intended wavelength (1 $50 \mathrm{~nm})$, which result in the "Quantum Size Effect" $[8,9]$. The energy level can be split into a series of values: $E_{1}, E_{2}, E_{3}, \ldots, E_{n}$.

3) The strain may result in the overall movement of the bottom and top of conduction band, and the separation of heavy and light holes.

As shown in Figure 1, the potential well material InGaAs, and the barrier materials AlGaAs and GaAsP were respectively used to prepare the strained quantum wells and strain compensated quantum wells. As for the strained quantum well, the barrier band gap structure will not vary with strain changes of the potential well. As the lattice matches with the substrate, the barrier can grow to a very large thickness, which has reduced the difficulty of the epitaxial growth. As for the strain compensated quantum wells, the introduction of the GaAsP material which does not match the substrate lattice may result in the restriction of the grown thickness. In order to reduce the lattice defects resulting from the dismatch, our $\mathrm{Al}$ and $\mathrm{P}$ components are 0.1 with the thickness of $20 \mathrm{~nm}$.

According to the literature [10] calculation method, we use the Kohn-Luttinger Hamiltonian to solve the quantum wells strain effect. By adopting this approach, we need to solve a $4 \times 4$ matrix. Through unitary transformation, we need to solve a $2 \times 2$ matrix calculation. By adopting the transfer matrix method, we can get a credible theoretical solution.

$$
H=\left[\begin{array}{cc}
H^{U} & 0 \\
0 & H^{L}
\end{array}\right]
$$

Wherein, the $H^{U}$ and $H^{L}$ are both $2 \times 2$ matrix.

As for the strained quantum well, the Hamiltonian is:

$$
\vec{H}=\left(\left[\begin{array}{cc}
P+Q+\zeta & R \\
R^{\dagger} & P-Q-\zeta
\end{array}\right]+V_{h}(z)\right)
$$

Wherein, 


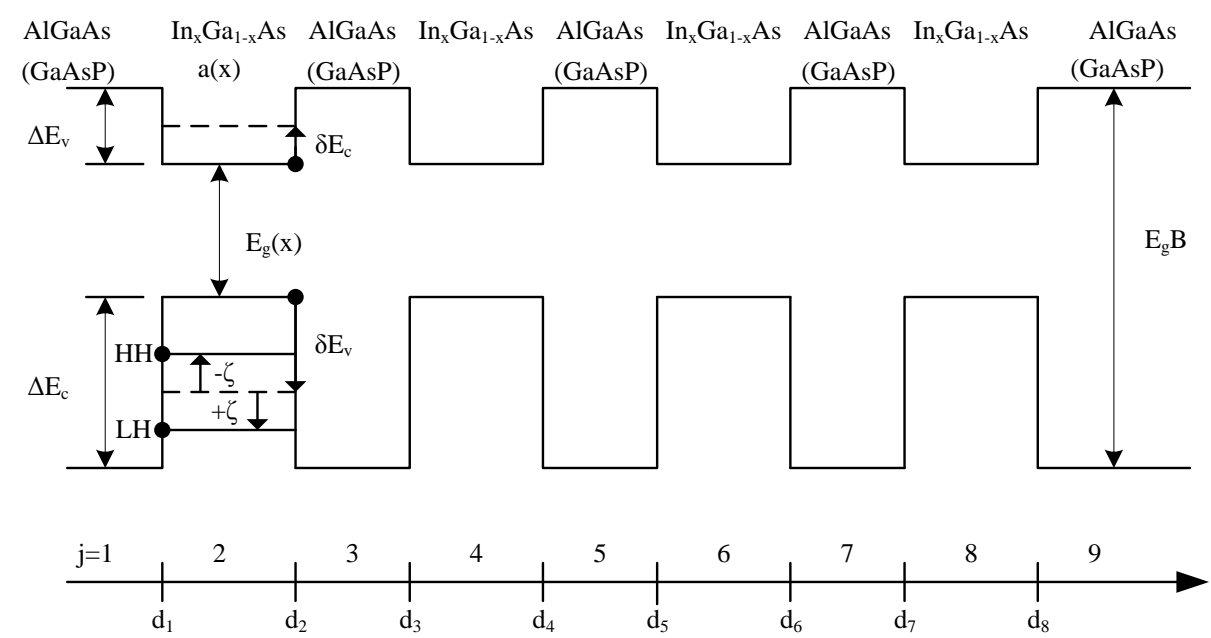

Figure 1. The energy-band diagram in real space.

$$
V_{h}(z)=\left\{\begin{array}{c}
2 a_{v}\left(1-\frac{C_{12}}{C_{11}}\right) \varepsilon \text { in the well } \\
\Delta E_{v} \text { in the barriers }
\end{array}\right.
$$

Through analysis of the continuous wave function, we use the matrix $U_{j(j+1)}$ to represent the relationship between adjacent layers,

$$
\left[\begin{array}{l}
A_{H H j} \\
B_{H H j}
\end{array}\right]=U_{j(j+1)}\left[\begin{array}{l}
A_{H H(j+1)} \\
B_{H H(j+1)}
\end{array}\right]
$$

By adopting the transfer matrix method, we obtained the $U_{t}$, and then the solution of $U_{t}$ can be gotten,

$$
\begin{gathered}
{\left[\begin{array}{l}
A_{H H 1} \\
B_{H H 1}
\end{array}\right]=U_{12} U_{23} \cdots U_{j(j+1)}\left[\begin{array}{l}
A_{H H(j+1)} \\
B_{H H(j+1)}
\end{array}\right]} \\
U_{t}=U_{12} U_{23} \cdots U_{j(j+1)}=\left[\begin{array}{ll}
u_{11} & u_{12} \\
u_{21} & u_{22}
\end{array}\right]
\end{gathered}
$$

Wherein,

$$
\begin{gathered}
U_{j(j+1)}=\frac{1}{2}\left[\begin{array}{cc}
1+p_{j(j+1)} & 1-p_{j(j+1)} \\
1-p_{j(j+1)} & 1+p_{j(j+1)}
\end{array}\right]\left[\begin{array}{cc}
e^{-i k_{(i+1) z} l_{j+1}} & 0 \\
0 & e^{i k_{(i+1) z} l_{j+1}}
\end{array}\right] \\
p_{j(j+1)}=\frac{m_{j} k_{(j+1) z}}{m_{j+1} k_{j z}} \\
k_{z}^{2}=\frac{1}{\frac{h^{2}}{2 m}\left(\gamma_{1}+\gamma_{2}\right)}\left[\bar{E}+\zeta-V_{h}(z)\right]
\end{gathered}
$$

By calculating the solution of $u_{11}=0$, we obtained $E$.

As the existence of the quantum size effect, we got a series of solutions: $E_{1}, E_{2}, E_{3}, \ldots, E_{n}$. They are corresponding to the different energy levels. The boundary condition determines that they can not exceed the poten- tial barrier height. The fact of the energy splitting resulting from the strain was also verified theoretically. As shown in Figures 2, and 3, the a, b, c in Figure 2 are the change trend of the wavelength along with the In composition and well width, which respectively represents the change trend of the wavelength of the strained quantum well conduction band energy, the amount of heavy-hole band, and between the nearest energy levels. Figure 3 shows the corresponding strain compensated quantum wells. By comparison, we found that the change trend of the energy band of the barrier material adopting the $\mathrm{Al}_{0.1} \mathrm{Ga}_{0.9} \mathrm{As}$ and $\mathrm{Ga}_{0.9} \mathrm{AsP}_{0.1}$ is consistent with the change trend of the transition wavelength.

As for the strain compensated quantum wells, when the thickness of the well layer $(8 \mathrm{~nm})$ is the same with the In component (0.2). Compared with the strained quantum well, the compensated quantum wells have introduced the tensile stress in the barrier material. The tensile stress served as Interaction force and acted on the potential well layer. The additional stress caused the reduction of the effective mass of the heavy hole. Due to the reduction of the heavy hole effective mass, the variation volume of the band gap resulting from the quantum size effect may increase, which finally resulting in an increase of the effective band gap and the blue shift of the wavelength. We can clearly find that this phenomenon in the following experiment. As the theoretical analysis model is a bit complex, we only conduct qualitative analysis here. We can also see the properties in the researches made by Duraev et al. [11].

\section{Results and Analysis}

After the text edit has been completed, the paper is ready for the template. Duplicate the template file by using the Save As command, and use the naming convention prescribed by your journal for the name of your paper. In 

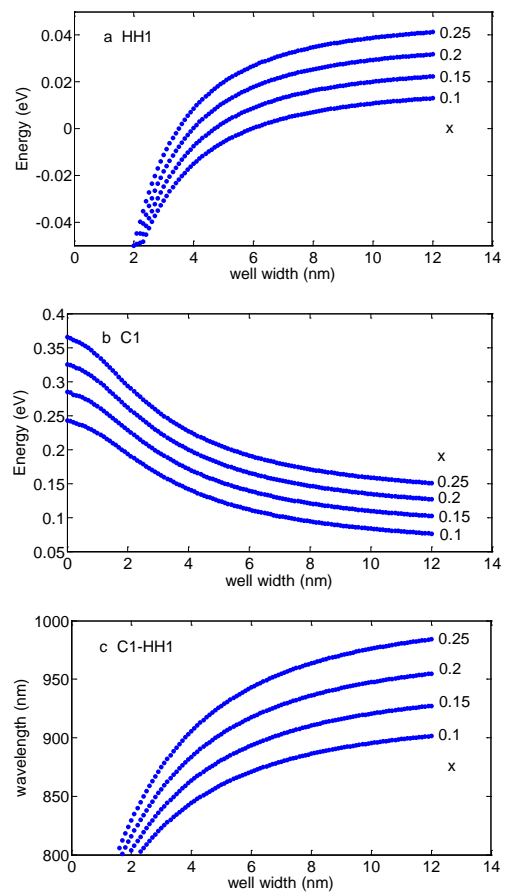

Figure 2. Change of the InGaAs/GaAsP strained quantum well energy following the In-group and the potential well width. $a$ is the first heavy-hole band; $b$ is the first conduction band, $c$ is the energy level transition wavelengths between the first heavy-hole band and the conduction band.
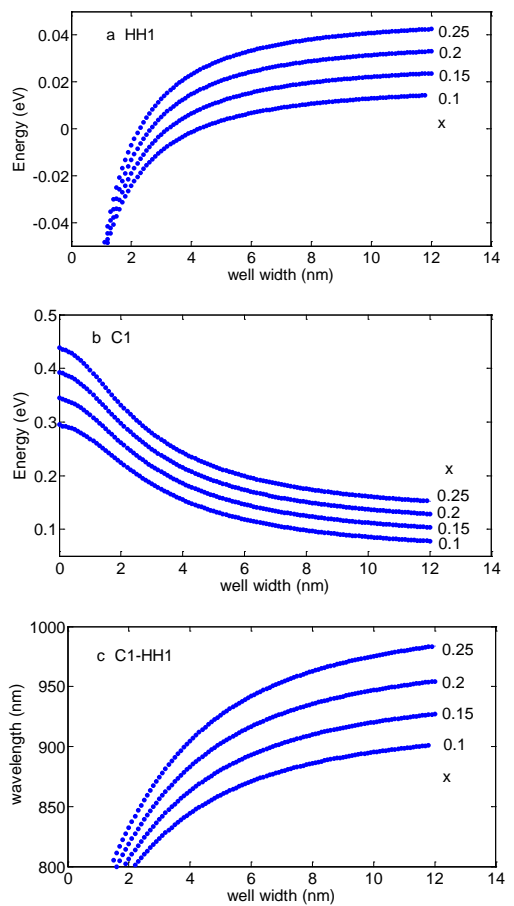

Figure 3. Change of the InGaAs/GaAsP strained compensated quantum well energy following the In-group and the potential well width. $a$ is the first heavy-hole band; $b$ is the first conduction band, $c$ is the energy level transition wavelengths between the first heavy-hole band and the conduction band. this newly created file, highlight all of the contents and import your prepared text file. You are now ready to style your paper.

Under the same environmental conditions (300 K), we conducted unified tests for the four samples. The test equipment is the Philips PLM100 type PL test system. Test results are shown in Table 3 . The In flow of sample 1 is $126.7 \mathrm{~cm}^{3}$, the corresponding component is 0.2 , and the luminescence center wavelength is $962 \mathrm{~nm}$. Because of the parameter control error resulting from the laboratory equipment, compared with the theoretical results, there is still a certain wavelength deviation.

The above theoretical studies have shown that the central wavelength is mainly affected by three parts: First, the InGaAs material changes resulting form the In composition. As the decrease of the In composition, the band gap of the quantum well material may increase; Second, due to the change of the stress, the conduction band will increase and the light and heavy holes may separate, and the band gap may change. As the increase of the In composition, the InGaAs lattice constant may increase, the well material stresses may also increase, the bottom of the conduction band and the top of valence band may decrease, the heavy and light hole may separate, the band gap may increase, and the wavelength may have a blue shift; Third, the narrow quantum well may result in width effect as well as a energy level split of the potential well.

Through study of the No 1 and No 3 samples, we can conduct qualitative analysis of the impact of the stress on the quantum wells. Sample No. 1 and No 3 are of the same In flow rate: $126.7 \mathrm{~cm}^{3}$. The No 3 Sample is the InGaAs/GaAsP strain compensated quantum wells. As the lattice constant of the $\mathrm{GaAs}_{0.9} \mathrm{P}_{0.1}$ barrier (5.6331 $\AA$ ) is less than the lattice constant of the GaAs substrate (5.6533 $\AA$ ), the barrier layer will subject to tensile strain. The tensile strain stress, as the interaction force, will act on the InGaAs well layers. The InGaAs well layers are inherently subjected to the compressive stress. The opposite stress of the barrier and the well compensate each other, to promote more uniform stress, which can greatly improve the stability of the quantum well. Due to the increase of the internal stress, the separation of light and heavy hole band may also increase. After comparison with the strained quantum well, we find that the center wavelength has a blue shift. From Figure 4, we can

Table 3. Measurements of the four samples of photoluminescence (PL).

\begin{tabular}{cccc}
\hline Sample & $\begin{array}{c}\text { Peak wavelength } \\
(\mathrm{nm})\end{array}$ & $\begin{array}{c}\text { Light intensity } \\
\left(\mathrm{AU}^{*}\right)\end{array}$ & $\begin{array}{c}\text { Half peak width } \\
(\mathrm{nm})\end{array}$ \\
\hline 1 & 962.0 & 3176.1 & 22.1 \\
2 & 928.0 & 3870.8 & 20.1 \\
3 & 952.0 & 4571.4 & 23.5 \\
4 & 912.0 & 4986.4 & 18.2 \\
\hline
\end{tabular}




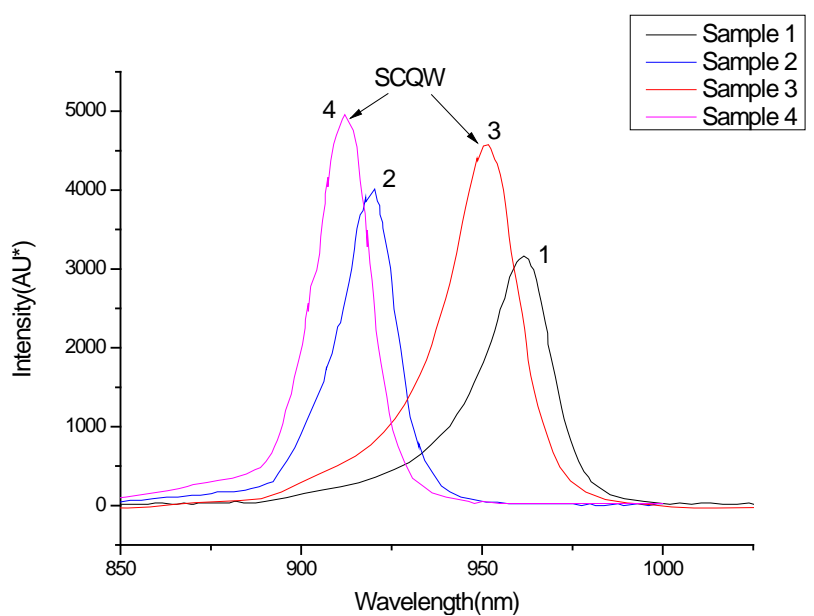

Figure 4. Photoluminescence of four samples.

clearly see that the No. 3 sample has an obvious blue shift compared with No 1 , reaching $10 \mathrm{~nm}$.

Based on the study of the four samples, we find that as the In composition decreases, the strained quantum wells and the strain compensated quantum wells, the peak halfwidth may reduce accordingly. As the decrease of the In component, the decrease of the lattice fit of the quantum well, the decrease of the corresponding stress, the decrease of the defects in the epitaxial growth process, and the increase of the flatness of the epitaxial layer, the light intensity can be effectively improved and the peak half width can be reduced. Therefore, the growth of the low In composition can improve the epitaxial growth of the active region.

Affect the optical properties of quantum wells factors on the one hand the strained quantum well type, the epitaxial growth process on the other hand.

After comparison of the No 1 and No 3 sample and comparison of the No 2 and No 4 sample, we found that the fluorescence intensity of the strain compensated quantum wells can be significantly improved on the basis of strained quantum well. This kind of improvement can not be fully explained by the strain mechanism. The strained quantum well barrier materials adopt the AlGaAs. As the $\mathrm{Al}$ is of high chemical activity, it can easily react with $\mathrm{O}$ impurities and then generate the Al-O bond. The incorporation of the $\mathrm{O}$ impurities may lead to the non-radiative recombination centers, which can greatly reduce the fluorescence intensity [12]. In contrast, the strain compensated quantum well adopts the GaAsP materials. The application of materials without $\mathrm{Al}$ can reduce the incorporation of the $\mathrm{O}$ impurities, which can effectively improve the fluorescence intensity.

\section{Summary}

We prepared four kinds of samples under the same con- ditions. The theoretical calculation and the experimental demonstration have verified the affecting factors of the characteristics of luminescence for the quantum well. This paper has focused on analysis of the stress on the optical properties of quantum wells. Due to the compressive stress, the wavelength of the fluorescence luminescence center may have a blue shift. The adoption of the growth of the In composition, the stress of the potential well layer may decrease, which may greatly improve the epitaxial flatness of the well layer. The barrier material adopts the GaAsP material, and InGaAs strain compensated quantum well structure is prepared. The stress compensation and properties of the without $\mathrm{Al}$ can greatly increase the optical properties of quantum wells.

\section{REFERENCES}

[1] J. V. Moloney, J. Hader and S. W. Koch, “Quantum Design of Semiconductor Active Materials Laser and Amplifier Applications,” Laser \& Photonics Reviews, Vol. 1, 2007, pp. 24-43. http://dx.doi.org/10.1002/lpor.200610003

[2] J. Hader, J. V. Moloney, M. Fallahi, et al., "Closed-Loop Design of a Semiconductor Laser," Optics Letters, Vol. 31, No. 22, 2006, pp. 3300-3302. http://dx.doi.org/10.1364/OL.31.003300

[3] H. Toktamis, B. Gonul and M. Oduncuoglu, “Comparative Study of the Band-Offset Ratio of Conventionally Strained and Strain Compensated InGaAs/GaAs QW Lasers,” Physica E, Vol. 24, 2004, pp. 183-186. http://dx.doi.org/10.1016/j.physe.2004.03.020

[4] N. K. Dutta, W. S. Hobson, D. Vakhshoori, et al., "Strain Compensated In-GaAs-GaAsP-InGaP Laser,” IEEE Photonics Technology Letters, Vol. 8, No. 7, 1996, pp. 852854. http://dx.doi.org/10.1109/68.502248

[5] A. Moser, A. Oosenbrug, E. E. Latta, Th. Forster and M. Gasser, "High-Power Operation of Strained InGaAs/AlGaAs Single Quantum Well Lasers,” Applied Physics Letters, Vol. 23, 1991, pp. 2642-2644. http://dx.doi.org/10.1063/1.105924

[6] P. K. Bhattacharya, "Semiconductor Optoelectronic Devices,” Pearson Education, US, 1996.

[7] R. M. Kolbas, N. G. Anderson, W. D. Ladig, et al., IIEEE Journal of Quantum Electronics, QE, Vol. 24, No. 8, 1998, p. 1605.

[8] S. L. Chuang, "Physics of Optoelectronic Devices," Wiley, New York, 1995.

[9] L. A. Coldren and S. W. Corzine, "Diode Lasers and Photonic Integrated Physics,” Wiley, New York, 1995.

[10] S. L. Chuang, "Efficient Band-Structure Calculations of Strained Quantum Wells,” Physical Review, Vol. B43, 1991, pp. 9649-9661.

[11] V. P. Duraev, A. A. Mamalyuk, A. A. Padalitsa, et al., "Effect of GaAsP Barrier Layers on the Parameters of InGaAs/AlGaAs Laser Diodes Emitting in the 1050 1100 nm Spectral Range,” Quantum Electronics, Vol. 35, No. 
10, 2005, pp. 909-911.

http://dx.doi.org/10.1070/QE2005v035n10ABEH005742

[12] D. C. Lu and S. K. Duan, "Principles and Applications of
Metal Organic Vapor Phase Epitaxy,” Science Press, Beijing, 2009, pp. 160-164. (in Chinese) 